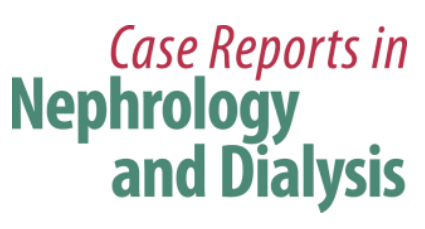

\title{
A Case of IgG2 Heavy Chain Deposition Disease in a Patient with Kappa Positive Plasma Cell Dyscrasia
}

\author{
Li Zhang $^{\mathrm{a}}$ Sindhuja Marupudi ${ }^{\mathrm{a}}$ Tamil Kuppusamy ${ }^{\mathrm{a}}$ William LaRosa $^{\mathrm{a}}$ \\ Paul Turer $^{a} \quad$ Meyer Heyman ${ }^{a}$ Lois Arend ${ }^{b}$ \\ ${ }^{a}$ St. Agnes Hospital, and ${ }^{b}$ Johns Hopkins University School of Medicine, Baltimore, \\ Md., USA
}

\section{Key Words}

Monoclonal gammopathy · Light chain · Immunoglobulin G

\begin{abstract}
IgG2 heavy chain deposition disease (HCDD) is extremely rare. To date, only 4 cases have been reported in medical literature. To our knowledge, we report the first case of IgG2 HCDD accompanied by kappa plasma cell dyscrasia.

(c) 2014 S. Karger AG, Basel
\end{abstract}

\section{Introduction}

Heavy chain deposition disease (HCDD) is a subtype of monoclonal immunoglobulin deposition disease and is characterized by glomerular, tubular, and vascular wall deposition of monoclonal heavy chains without associated light chain deposition [1]. More than two decades after Aucouturier et al. [2] described the first 2 cases, HCDD remains an uncommon condition [3-6], with fewer than 40 cases reported [7]. The rarity of the disease, the unknown natural history, and the lack of evidence-based guidelines for treatment, make HCDD challenging to treat.

Thus far, the reported cases of HCDD have involved the $\gamma 1-4, \alpha$, and $\mu$-heavy chain subtypes [7]. Cases of $\gamma 2$-HCDD are extremely rare, with only 4 cases reported in the literature to date $[3,4,8,9]$. The most common pathological finding of HCDD is nodular glomerular sclerosis; however, glomerular crescentic formation has also been described. Membranoproliferative glomerulonephritis (MPGN) and intracapillary proliferative glomerulonephritis are less common $[3,6,10]$. We describe a case of $\gamma 2$-HCDD with kappa plasma cell dyscrasia and unique kidney biopsy findings.

Lois J. Arend, MD, PhD

Department of Pathology

Johns Hopkins University School of Medicine

720 Rutland Ave., Ross 632E, Baltimore, MD 21205 (USA)

E-Mail ljarend@jhu.edu 


\section{Case Reports in \\ Nephrology and Dialysis}

\begin{tabular}{l|l}
\hline Case Rep Nephrol Dial 2015;5:6-12 & \\
\hline DOI: 10.1159/000366053 & $\begin{array}{l}\text { @ 2014 S. Karger AG, Basel } \\
\text { www.karger.com/cnd }\end{array}$ \\
\hline
\end{tabular}

Zhang et al.: A Case of IgG2 Heavy Chain Deposition Disease in a Patient with Kappa Positive Plasma Cell Dyscrasia

\section{Case Report}

\section{Clinical History and Laboratory Data}

A 56-year-old previously healthy Caucasian female presented with edema, hypertension, and rapidly progressive renal failure. Three months prior to presentation, the patient's serum creatinine was $1.9 \mathrm{mg} / \mathrm{dl}$. At the time of admission, her creatinine was $8 \mathrm{mg} / \mathrm{dl}$. Urinalysis showed protein (2+), red blood cells (2+), and her 24-hour urine protein was 11.6 grams. In addition to having a normocytic normochromic anemia, her serum IgG was 372 $\mathrm{mg} / \mathrm{dl}(694-1,618)$, free kappa light chain (LC) was $339.0 \mathrm{mg} / \mathrm{dl}(0.33-1.94)$, free lambda LC was $5.91 \mathrm{mg} / \mathrm{dl}(0.57-2.63)$, and the kappa/lambda ratio was 57.36 (0.26-1.65). Serum IgA, IgM, C3, and C4 were normal. Urine immunofixation showed kappa $275 \mathrm{mg} / \mathrm{dl}(0.14-2.42)$, lambda $5.93 \mathrm{mg} / \mathrm{dl}$ (0.02-0.67), and 24-hour urine kappa light chain excretion of 4.3 grams. ANA, antineutrophil cytoplasmic antibody, anti-GBM, cryoglobulin, hepatitis B surface antigen and HIV were all negative. Notably, her hepatitis C virus (HCV) antibody was positive, but HCV was undetectable by PCR. Renal ultrasound showed normal sized kidneys with moderately increased bilateral renal echogenicity. A bone survey showed no lytic lesions. Bone marrow biopsy revealed an abnormal kappa positive plasma cell population (CD138-positive) representing 5\% or less of the cellularity (fig. 1a, b). Flow cytometry confirmed an abnormal CD56+ plasma cell population with an elevated kappa/lambda ratio of 6.2 , suggestive of a plasma cell neoplasm.

\section{Kidney Biopsy}

On the kidney biopsy, 37 glomeruli were present, 7 of which were obsolescent. Ten glomeruli had cellular to fibrocellular crescents (fig. 1c), with focal fibrinoid necrosis (fig. 1d). Most glomeruli exhibited global proliferation, with mesangial and endocapillary hypercellularity as well as lobular accentuation. Double contour formation of the glomerular basement membrane was frequently seen (fig. 1e). Congo red staining for amyloid was negative.

Direct immunofluorescence staining showed 4+ smudgy to coarse granular mesangial, segmental linear glomerular capillary wall (fig. 2a), as well as diffuse linear tubular basement membrane, and arterial wall staining for IgG (fig. 2b). IgG subclass staining demonstrated 2-3+ deposition in these locations only for IgG2 (fig. 2c), with faint staining of mesangial nodules for IgG4. Both IgG1 and IgG3 staining were negative. Staining for light chains was negative for lambda, with trace to $1+$ for kappa in expanded mesangial regions and scant staining of basement membranes (fig. 2d). Staining for C3 was 3+ in the glomerular mesangium, segmental glomerular capillary walls, and focal artery and arteriole walls. Tubular casts stained 2-3+ for IgA, kappa, and lambda.

Electron microscopy revealed significant effacement of podocyte foot processes. The mesangial regions were moderately expanded by matrix and contained finely granular electron dense material (fig. 2e) with vague fibrils in a few segments (fig. 2f). A band of finely granular electron dense material was present along some glomerular basement membranes and in rare tubular basement membranes (fig. $2 \mathrm{~g}$, h).

\section{Diagnosis}

The pathological findings in this case were consistent with IgG2 heavy chain monoclonal immunoglobulin deposition disease. In two previous studies [1, 3], the following criteria were used for the diagnosis of HCDD: staining for a single class of $\operatorname{Ig}(\gamma, \alpha, \mu)$ on glomerular and/or tubular basement membranes, negative or minimal staining for light chain ( $\kappa$ or $\lambda$ ), and typical finely granular basement membrane-dominant electron dense deposits. 


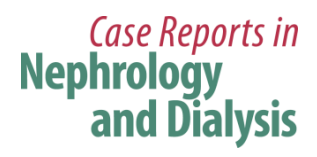

Case Rep Nephrol Dial 2015;5:6-12

DOI: $10.1159 / 000366053$

2014 S. Karger AG, Basel

www.karger.com/cnd

Zhang et al.: A Case of IgG2 Heavy Chain Deposition Disease in a Patient with Kappa Positive Plasma Cell Dyscrasia

Although Aucouturier et al. [2] suggested the hallmark of HCDD is CH1 deletion of $\gamma$-heavy chains, staining for $\mathrm{CH} 1$ is not necessarily required for a diagnosis [7]. The present case meets the criteria for $\gamma 2$-HCDD, making it the fifth case reported in the literature to our knowledge. Light microscopy demonstrated a general pattern consistent with MPGN; however, the presence of crescents and fibrin are unusual for many conditions that result in an MPGN pattern. The differential diagnosis was broad, encompassing diseases that produce an MPGN pattern and/or crescentic disease, including: MPGN type 1, C3 glomerulopathy, proliferative glomerulonephritis with monoclonal immunoglobulin deposits, fibrillary glomerulopathy, and autoimmune diseases. Given the proliferative pattern, antineutrophil cytoplasmic antibody-associated small vessel vasculitis and anti-GBM glomerulonephritis were less likely. Immunofluorescence demonstrated an immune complex-associated process related to the deposition of IgG2 molecules that did not appear to be associated with significant light chain deposits. The Congo red staining was negative. While some areas of expanded mesangium contained vague fibrillar structures, they were not typical of amyloid, were slightly thinner than expected for fibrillary glomerulopathy, and lacked classic features for cryoglobulin. The lack of significant associated kappa light chain deposits in the present case was surprising, given the large quantity in serum and urine.

The patient's renal function did not improve despite pulsed steroids, followed by prednisone and oral cyclophosphamide, and therefore, hemodialysis was started. Three months into treatment, her serum kappa/lambda ratio decreased dramatically to 2.2; however, the patient remains dialysis-dependent. Recently, her treatment regimen has been changed to weekly bortezomib, cyclophosphamide and dexamethasone.

\section{Discussion}

Patients with HCDD often present with nephrotic syndrome, hypertension, and progressive renal failure. Monoclonal gammopathy is frequently observed, but it is not a requirement for the diagnosis. Hypocomplementemia is found in most cases of $\gamma 1-$ and $\gamma 3-$ HCDD [11], but it is not typical of $\gamma 2$-HCDD, as the IgG2 molecule does not bind complement as effectively [12]. In HCDD, free heavy chains are often not detected in the serum due to their high affinity for tissues, which results in low serum concentration. Serum fractionation followed by immunoblotting is more likely to detect monoclonal heavy chains lacking CH1 epitopes in whole serum samples compared to Western blot analysis [13]. However, these assays are not readily available in clinical practice.

Oe et al. [7] have recently reviewed HCDD and found that 24\% (9/37) of patients had a hematological malignancy (MM 7, plasmacytoma 1, amyloidosis 1), and 70\% (26/37) of cases had a detectable serum $M$ protein. Komatsuda et al. [14] reported a case of $\gamma 1$-HCDD that initially responded to corticosteroid therapy, but 10 years later, the patient developed AL amyloidosis and died of congestive heart failure. Thus, HCDD may be a precursor of more advanced plasma cell malignancy, warranting hematology referral and long-term follow-up. Patients with HCDD may benefit from chemotherapy on the basis of the MM regimen to prevent mortality and morbidity from other organ damage, even if the kidney failure is not reversible. The prognosis of HCDD is very poor. One-third to one-half of patients became hemodialysis dependent within 1 year, and approximately 10\% of patients died within 6 years. Lower serum creatinine level as well as early diagnosis and treatment are associated with better outcomes [7].

As seen in our case, many patients with HCDD have positive HCV antibody without detectable HCV-PCR. There is growing evidence suggesting that HCV is associated with B-cell 
Zhang et al.: A Case of IgG2 Heavy Chain Deposition Disease in a Patient with Kappa Positive Plasma Cell Dyscrasia

lymphoproliferative disorders [15], and undetectable serum HCV-PCR cannot rule out the presence of viral DNA in other tissues [16]. However, this could be a result of false-positive tests due to the cross reaction between HCV immunoassay and the abnormal heavy chain or a spontaneous remission of HCV infection [1]. The association between HCV and HCDD deserves further investigation.

\section{Acknowledgements}

We would like to thank our patient for giving consent of the case material for this publication.

\section{Disclosure Statement}

The authors declare no conflicts of interest.

\section{References}

$\checkmark 1$ Lin J, Markowitz GS, Valeri AM, et al: Renal monoclonal immunoglobulin deposition disease: the disease spectrum. J Am Soc Nephrol 2001;12:1482-1492.

2 Aucouturier P, Ahmed K, Touchard G, et al: Brief report: heavy-chain deposition disease. N Engl J Med 1993;329:1389-1393.

-3 Nasr SH, Valeri AM, Cornell LD, et al: Renal monoclonal immunoglobulin deposition disease: a report of 64 patients from a single institution. Clin J Am Soc Nephrol 2012;7:231-239.

4 Herzenberg AM, Kiaii M, Magil AB: Heavy chain deposition disease: recurrence in a renal transplant and report of IgG(2) subtype. Am J Kidney Dis 2000;35:E25.

5 Fogo AB, Rossini M: Monoclonal immunoglobin deposition disease, heavy chain deposition type. Am J Kidney Dis 2003;42:A48, E1-2.

-6 Vedder AC, Weening JJ, Krediet RT: Intracapillary proliferative glomerulonephritis due to heavy chain deposition disease. Nephrol Dial Transplant 2004;19:1302-1304.

7 Oe Y, Soma J, Sato H, et al: Heavy chain deposition disease: an overview. Clin Exp Nephrol 2013;17:771-778.

-8 Guiard E, Karras A, Plaisier E, et al: Patterns of noncryoglobulinemic glomerulonephritis with monoclonal Ig deposits: correlation with IgG subclass and response to rituximab. Clin J Am Soc Nephrol 2011;6:16091616.

$\$ 9$ Chen GT, Liao XH, Yan RY, et al: A case of renal $\gamma 2$ heavy-chain deposition disease accompanied by rapid progressive renal failure. Ir J Med Sci 2014;183:319-321.

10 Alexander MP, Nasr SH, Watson DC, et al: Renal crescentic alpha heavy chain deposition disease: a report of 3 cases and review of the literature. Am J Kidney Dis 2011;58:621-625.

11 Soma J, Sato K, Sakuma T, et al: Immunoglobulin gamma3-heavy-chain deposition disease: report of a case and relationship with hypocomplementemia. Am J Kidney Dis 2004;43:E10-E16.

$\checkmark 12$ Tao MH, Smith RI, Morrison SL: Structural features of human immunoglobulin G that determine isotypespecific differences in complement activation. J Exp Med 1993;178:661-667.

13 Moulin B, Deret S, Mariette X, et al: Nodular glomerulosclerosis with deposition of monoclonal immunoglobulin heavy chains lacking C (H) 1. J Am Soc Nephrol 1999;10:519-528.

14 Komatsuda A, Maki N, Wakui H, et al: Development of systemic lambda-light chain amyloidosis in a patient with gamma-heavy chain deposition disease during long-term follow-up. Nephrol Dial Transplant 2005;20:434-437.

-15 Mollejo M, Menárguez J, Guisado-Vasco P, et al: Hepatitis C virus-related lymphoproliferative disorders encompass a broader clinical and morphological spectrum than previously recognized: a clinicopathological study. Mod Pathol 2014;27:281-293.

16 Zignego AL, Macchia D, Monti M, et al: Infection of peripheral mononuclear blood cells by hepatitis C virus. J Hepatol 1992;15:382-386. 


\section{Case Reports in \\ Nephrology \\ and Dialysis}

\begin{tabular}{l|l}
\hline Case Rep Nephrol Dial 2015;5:6-12 & \\
\hline DOI: 10.1159/000366053 & $\begin{array}{l}\text { @ 2014 S. Karger AG, Basel } \\
\text { www.karger.com/cnd }\end{array}$ \\
\hline
\end{tabular}

Zhang et al.: A Case of IgG2 Heavy Chain Deposition Disease in a Patient with Kappa Positive Plasma Cell Dyscrasia
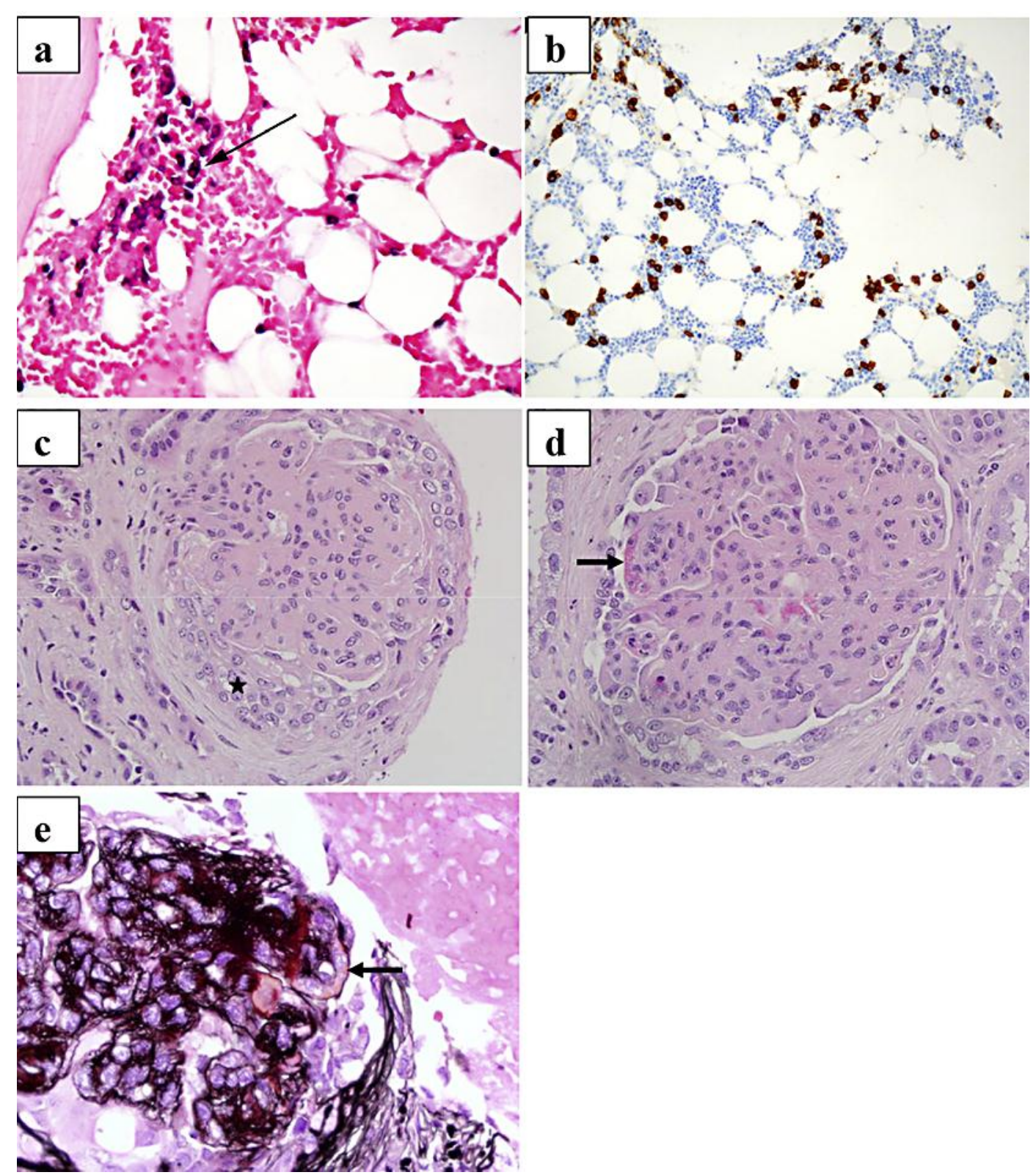

Fig. 1. Bone marrow (a, b) and kidney (c-e) biopsies, light microscopy. a In situ hybridization for kappa light chain, with a positive signal indicated by blue staining (arrow). b Immunohistochemistry for plasma cells (CD138). c Lobulated glomerulus with extracapillary proliferation (crescent; star; HE). d Hypercellular glomerulus with segmental fibrinoid necrosis (arrow; HE). e Duplication of glomerular basement membrane (arrow; Jones silver). 
Case Reports in

Nephrology

and Dialysis
Case Rep Nephrol Dial 2015;5:6-12

DOI: $10.1159 / 000366053$

Zhang et al.: A Case of IgG2 Heavy Chain Deposition Disease in a Patient with Kappa Positive Plasma Cell Dyscrasia
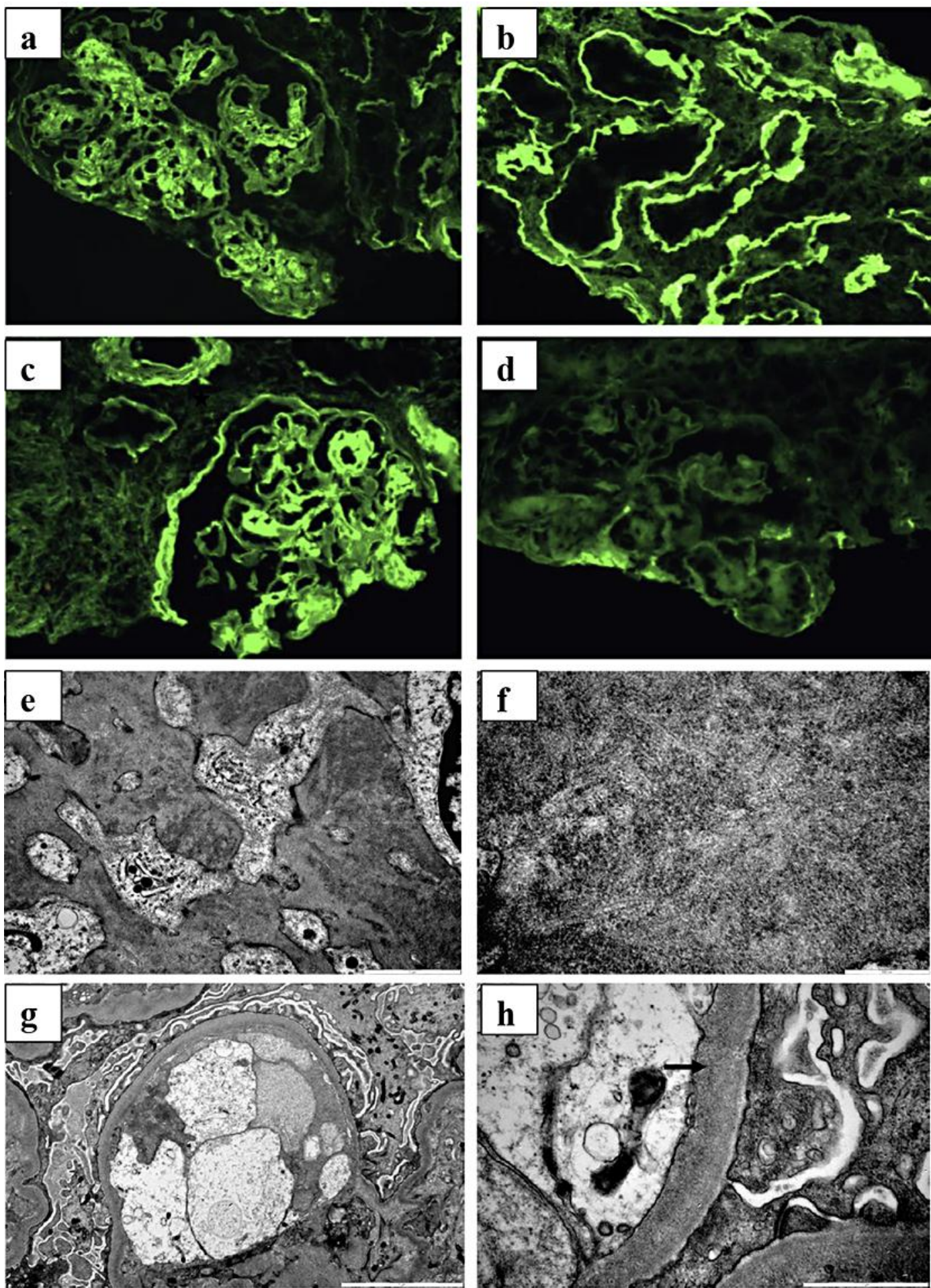

Fig. 2. Kidney biopsy. a-d immunofluorescence microscopy, $\mathbf{e}-\mathbf{h}$ electron microscopy. a IgG staining of glomerular mesangial regions and segmental glomerular capillary walls. b Linear IgG staining of tubular basement membranes. c IgG2 subclass staining of glomerular mesangium, segmental glomerular capillary walls and the small artery wall. $\mathbf{d}$ Faint staining for kappa light chain in expanded mesangial regions and scant staining of glomerular and tubular basement membranes. e Mesangial electron dense deposits. $\mathbf{f}$ Mesangial deposits with vague linear/fibrillar substructure. $\mathbf{g}, \mathbf{h}$ Glomerular capillary wall with finely 
Case Reports in

Nephrology

and Dialysis
Case Rep Nephrol Dial 2015;5:6-12

DOI: $10.1159 / 000366053$

2014 S. Karger AG, Base

www.karger.com/cnd

Zhang et al.: A Case of IgG2 Heavy Chain Deposition Disease in a Patient with Kappa Positive Plasma Cell Dyscrasia

granular electron dense material along the inner aspect (arrow). 